\title{
Synchronous Hodgkin Lymphoma and Papillary Thyroid Carcinoma: The Importance of Positron Emission Tomography/Computed Tomography (PET/CT)
}

Burak Uz ${ }^{1}$

${ }^{1}$ Adult Hematology Clinic, Medicana International Samsun Hospital, Samsun, Turkey

Correspondence:

Burak Uz, Assoc Prof.

Address: Yeni Mahalle, Şehit Mesut Birinci Caddesi, No: 85, postal code: 55080, Canik, Samsun, Turkey

Email: burakuz78@gmail.com
Received: 23.12.2018,

Accepted: 19.03.2019

https://doi.org/10.5799/jcei/5761
Dear Editor,

We report a 50-year-old male with synchronous Hodgkin lymphoma (HL) and papillary thyroid carcinoma (PTC). The patient was diagnosed as having HL of the nodular sclerosis type on excisional biopsy of an enlarged right scalene node in March 2018. After further examinations including bone marrow biopsy and positron emission tomography/computed tomography (PET/CT) with 2-[18F]-fluoro-2-deoxy-Dglucose (18F-FDG), he was classified as having stage IVA HL with bone marrow involvement. The patient's medical history was unremarkable for any chronic disease or previous throid disease. He had no history of irradiation to head and neck, nor had any regular medications. Her mother had a diagnosis of breast carcinoma.

He was treated with 4 cycles of doxorubicin, bleomycin, vinblastine, and dacarbazine (ABVD) followed by 2 cycles of AVD. Initial PET/CT revealed no uptake in thyroid gland. Interim PET/CT revealed a complete remission except an increased uptake (SUVmax: 8,5) in right thyroid nodule $(1,3 \times 1 \mathrm{~cm})$. At this time, throid function tests were in normal ranges and thyroid auto-antibodies including antithyroid peroxidase and anti-thyroglobulin were negative. A thyroid ultrasonography (USG) revealed 2-3 isoechoic heterogen nodules surrounded by hypoechogenic halo with a maximum diameter of $0,9 \mathrm{~cm}$. Postchemotherapy PET/CT detected the aforementioned nodule $(1,3 \times 1 \mathrm{~cm})$ with a SUVmax of 9,6. However, a repeated thyroid
USG showed a conglomerated isoechoic nodular appearance with lobulated contours having milimetric and microcalcifications with a size of $13 \times 11,5 \times 25 \mathrm{~mm}$ in the right upper zone. Fine needle aspiration biopsy (FNAB) was consistent with a suspected diagnosis of PTC. The patient underwent total thyroidectomy, and histopathologic examination demonstrated a $0.6 \mathrm{~cm}$ PTC classical variant in the left lobe, and a $1.5 \mathrm{~cm}$ PTC follicular variant in the right lobe. One out of two resected lymph nodes were positive. Vessel and nerve involvement was not detected. The patient started to receive thyroid hormone replacement $(100 \mathrm{mcg} \mathrm{L}-$ thyroxin, daily) and radioactive iodine therapy was planned.

In a previous study [1], 6 out of 1,677 HL patients (relative risk: 15.6) who underwent irradiation developed thyroid malignancy after a latent period of 9-19 years (median 13 years). Abboud et al. have reported a 24year-old woman who was diagnosed with PTC after 10 years of disease-free HL. This patient had undergone 6 cycles of ABVD for stage IA HL without external radiotherapy [2].

The incidence of thyroid incidentaloma detected by whole body 18F-FDG-PET/CT is quite variable $(0.2-8.9 \%)$ with a pooled incidence of $2.46 \%$. Diffuse (e.g. Grave's disease, thyroiditis) and most focal FDG uptakes (e.g. thyroid nodules) are commonly associated with benign disorders, but approximately more than one third of these lesions are pathologically proven to be 
malignant. PTC is the most common histologic type accounting for approximately $84 \%$ of malignant thyroid incidentalomas. Role of standardized uptake value (SUV) value in differentiating malign from benign lesions is undefinite. Some researchers have found statistically significant difference between SUV value of benign (range: 3.0-6.7, $\mathrm{p}<0.05$ ) and malign thyroid incidentalomas (range: 4.53-14.2, p <0.05). Moreover, it has been shown that utilization of pre-opereative PET/CT in thyroid nodules with indeterminate, repeteadly insufficient, or non-diagnostic FNAB results revealed efficacious sensitivity (75-100\%) and negative predictive value (77-100\%) rates [3]. In the present case, interim and post-therapy SUVmax values were considerably high (8.5 and 9.6, respectively) which were suggestive of a thyroid malignancy.

Although no SUVmax cutoff value can be considered safe to discriminate malign from benign thyroid incidentalomas, about one third of focal uptakes are malignant. Therefore, suspected thyroid gland lesions with high SUV values detected by FDG-PET scanning should be further evaluated in terms of primary thyroid carcinoma. Initial work-up may include thyroid function tests, thyroid auto-antibodies, a detailed thyroid USG, and a possible FNAB.

Declaration of interest: The authors report no conflicts of interest.

Financial Disclosure: No financial support was received.

\section{REFERENCES}

1. Hancock SL, Cox RS, McDougall IR. Thyroid diseases after treatment of Hodgkin's disease. N Engl J Med. 1991;325:599-605.

2. Abboud B, Yazbeck T, Daher R, Chahine G, Ghorra C. Papillary thyroid carcinoma after chemotherapy for Hodgkin's disease. Am Surg. 2010;76:1316-7.

3. Bertagna F, Treglia G, Piccardo A, Giubbini R. Diagnostic and clinical significance of F-18-FDGPET/CT thyroid incidentalomas. J Clin Endocrinol Metab. 2012;97:3866-75. 